\title{
IDENTIFYING BIOLOGICAL PATHWAYS VIA PHASE DECOMPOSITION AND PROFILE EXTRACTION
}

\author{
Yi Zhang and Zhidong Deng* \\ Department of Computer Science, Tsinghua University \\ Beijing, 100084, China \\ *Email: michael@tsinghua.edu.cn
}

\begin{abstract}
Biological processes are always carried out through large numbers of genes (and their products) and these activities are often organized into different cellular pathways: sets of genes that cooperate to finish specific biological functions. Owing to the development of microarray technology and its ability to simultaneously measure the expression of thousands of genes, effective algorithms to reveal biologically significant pathways are possible. However, some open problems such as large amount of noise in microarrays and the fact that most biological processes are overlapping and active only on partial conditions pose great challenges to researchers. In this paper, we proposed a novel approach to identify overlapping pathways via extracting partial expression profiles from coherent cliques of clusters scattered on different conditions. We firstly decompose gene expression data into highly overlapping segments and partition genes into clusters in each segment; then we organize all the resulting clusters as a cluster graph and search coherent cliques of clusters; finally we extract expression profiles from coherent cliques and shape biological pathways as genes consistent with these profiles. We compare our algorithm with several recent models and the experimental results justify the superiorities of our approach: robustly identifying overlapping pathways in arbitrary set of conditions and consequently discovering more biologically significant pathways in terms of enrichment of gene functions.
\end{abstract}

\section{INTRODUCTION}

The rapid development of high-throughput techniques such as Oligonucleotide and cDNA microarrays [5] enable measuring the expression of thousands of genes simultaneously. This possibility offers an unprecedented opportunity to characterize the underlying mechanisms of a living cell. Activities of a living cell are so complex that different sets of genes participate in diverse biological processes to perform various cell functions. In this sense, identifying cellular pathways, sets of coherent genes that coordinate in biological processes to achieve specific functions, plays a considerable role in gaining an insight into the cell's activities.

Recently, researchers have made tremendous efforts to identify coherent gene groups [10]. Pioneering work includes agglomerative algorithm for hierarchical clustering [7], K-Means clustering of genes [17] and some graph-theoretical approaches for gene-based clustering [15]. Admittedly, applying traditional clustering algorithms on gene expression data can provide us with new perspectives on cellular processes. However, several problems in this process should be highlighted: (1) biological processes are active only on partial conditions. This fact renders clustering genes on entire conditions ineffective. (2) Extremely high noise exists in microarrays, which calls for robust models for pathway identification. (3) Partitioning genes into mutually exclusive clusters is unreasonable in that biological pathways are always overlapping.

Biclustering algorithms [14] are designed to capture biological processes active on part conditions. Different from traditional clustering methods, these models perform simultaneous clustering on both rows and columns and thus finally discover coherent submatrices where rows refer to genes and columns correspond to relevant conditions. One challenge to biclustering is that all the possible combinations of various genes and conditions are almost infinite.

Furthermore, overlapping property of cellular pathways is also mentioned by recent work. On one hand, some biclustering algorithms discover submatrices one after another and thus naturally yield non-exclusive biclusters. For instance, Cheng et al [6] mask the previous biclusters with random numbers and find other ones. Similarily, in [12] each bicluster merely deals with the "residual" expression of previous biclusters. On the other hand, algorithms aim to discover overlapping pathways simultaneously also existed. Battle et al [4] proposed a probabilistic model to discover overlapping biological processes concurrently.

Managing high noise in gene expression data is also indispensable for successfully determining

\footnotetext{
${ }^{*}$ Corresponding author.
} 
coherent genes. In [2], the author engages robust similarity measure based on the rank of expression in each condition, rather than the accurate expression level, to model the similarity between expression profiles of genes. This sort of measures is robust in the sense that they mainly focus on the rough shape of expression profile and will not be affected by disturbances on accurate expression level. The consensus clustering algorithm in [9] combine different clustering results to form a clustering ensemble. The underlying idea of this ensemble learning approach is that integrating opinions of different "experts" can yield a robust estimation.

In fact, an algorithm address all of these open problems is highly desirable. In this paper, we propose a strategy that satisfies all these demands: robustly discovering overlapping pathways on partial conditions. Other than traditional approaches that directly seek grouping over genes, our algorithm identifies cellular pathways by robustly searching expression profiles over arbitrary set of conditions. The key ideas of our approach are: (1) decompose the entire conditions into highly overlapping segments and clustering genes over each segment; (2) manage all resultant clusters into a cluster graph and discover coherent cliques on cluster graph; (3) extract expression profiles over coherent cliques and shape overlapping pathways according to the these profiles. As a result, this algorithm is capable of robustly recognizing overlapping molecular pathways on partial conditions and thus furnishing biologically significant sets of genes in terms of enrichment of gene functions.

\section{METHOD}

Our pathway discovery algorithm consists of three steps: (1) decomposing conditions into overlapping segments and performing gene clustering on each segment; (2) construct a cluster graph from the resulting clusters over all segments and discovering coherent cliques on the graph; (3) extracting expression profile from each coherent clique and identifying biological pathway according to each profile. In the rest of this section, we examine these steps in section 2.1 2.3 and analyze the properties of this algorithm in section 2.4.

\subsection{Phase Decomposition}

In order to capture biological processes in partial conditions, we divide the entire conditions (i.e. columns)

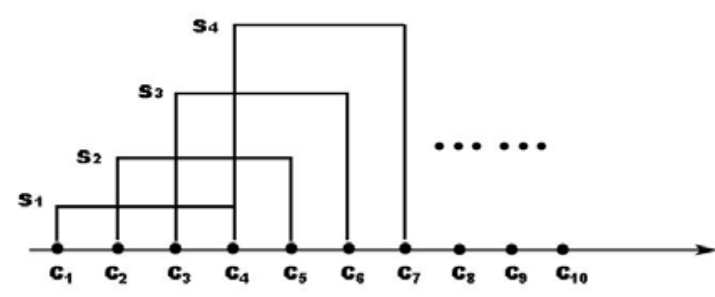

Fig. 1. Phase Decomposition

into highly overlapping segments. Each segment contains all the rows and a few columns in gene expression matrix. Then we discover co-expressed genes by gene-based clustering on each segment. Finally, large clusters are retained for later processing.

The first step is to decompose gene expression matrix into many segments. The goal of this decomposition is to ensure that biological process active on any partial conditions can be discovered by combining some segments. Note the term "segment" refers to a submatrix in gene expression matrix where all rows and a subset of columns are included. The decomposition strategy is shown in figure 1. Each segment covers fixed amount of conditions and advances a small step based on the previous segment. For instance, $\mathrm{s}_{1}$ covers $\left\{\mathrm{c}_{1}, \mathrm{c}_{2}, \mathrm{c}_{3}, \mathrm{c}_{4}\right\}$ and $\mathrm{s}_{2}$ contains $\left\{\mathrm{c}_{2}\right.$, $\left.c_{3}, c_{4}, c_{5}\right\}$. Two parameters are involved: (1) segment length $L$ : the number of conditions covered by a segment. Note that using too large $L$ loses the possibility to discover pathways active on short period; while engaging too small $L$ makes clustering on each segment ineffective: co-expression on very short period always appears by chance. We set $L=4$ in figure 1 only for illustration; such short segments will not be used in experiments. (2) step length $\Delta L$ : in figure 1 , we set $\Delta L$ $=1$, thus any biological process whose life-span is larger than $L$ can be obtained from combining certain segments. For instance, period $\mathrm{C}_{2} \sim \mathrm{C}_{6}$ can be captured by integrating two segments $s_{2}$ and $s_{3}$. One may choose larger step length in order to reduce the total number of segments. But fortunately, combinations of different segments can approximately represent any period larger than $L$, as long as segments are highly overlapping.

The second procedure is gene-based clustering on each segment so as to obtain the co-expression group. Here we use hierarchical clustering, with average link and Pearson correlation, to group genes on each segment. On each segment, cutting the hierarchical tree at specific threshold 1-c will produce many sets of co- 
expressed genes. Note $c$ is a key parameter of our algorithm: two gene expression profiles are considered coherent when their Pearson correlation is larger than $c$, i.e. their distance is smaller than 1-c.

At last, clusters which contain less than 5 genes are discarded in that too small clusters are considered outliers or biologically insignificant groups.

\subsection{Coherent Clique on Cluster Graph}

After clustering on each segment as discussed in section 2.1, we obtain many co-expression gene clusters. Clusters on the same segment are mutually exclusive while clusters computed from different segments may be highly overlapping, especially when step length is small and thus adjacent segments may present similar structure on gene expression. In this section, we address the problem of how to utilize these clusters to discover biological processes that active on arbitrary period. For this purpose, we propose the concepts of cluster graph and coherent clique; then we focus on how to discover coherent cliques on cluster graph. Note that searching coherent cliques is to find possible biological processes.

Firstly, given two gene clusters $C$ and $C$ ', we define the overlapping degree, and use this definition to offer a distance measure between clusters. Note that $|C|$ is the amount of genes in cluster $C$.

- $\operatorname{Overlap}\left(C, C^{\prime}\right)=\frac{\left|C \cap C^{\prime}\right|}{\left|C \cup C^{\prime}\right|}$

- $\operatorname{Distance}\left(C, C^{\prime}\right)=1-\operatorname{Overlap}\left(C, C^{\prime}\right)$

Secondly, after defining the distance between clusters, all clusters obtained from the procedures discussed in section 2.1 constitute a large cluster graph, which furnishes us with a global view of relationships among genes over different segments. Cluster graph $G(V, E)$ is a complete graph where each node $v \in V$ refers to a cluster $C$ and the weight of an edge $e=\left(v_{1}, v_{2}\right) \in E$ is the distance between two clusters corresponding to $v_{1}$ and $v_{2}$.

Thirdly, the concept of $\beta$-coherent clique is proposed as following: a $\beta$-coherent clique $Q\left(V^{\prime}, E^{\prime}\right)$ in a cluster graph $G(V, E)$ is a complete subgraph in $G$, satisfying that (1) any edge in $E$ ' has an weight less than $\beta$. (2) $V^{\prime}$ contains at least two nodes. Note that a $\beta$ coherent clique is biologically meaningful: (1) any two clusters in a coherent clique has a distance smaller than $\beta$, i.e. an overlapping degree larger than $1-\beta$; (2) clusters in a $\beta$-coherent clique must come from different segments, since clusters from same segment are mutually exclusive; (3) The fact that several clusters from diverse segments share a large proportion of common co-expressioned genes indicates the existence of a biological process which is active on the period composed of these segments.

Finally, given a cluster graph, we want to discover $\beta$-coherent cliques on this graph. An effective algorithm to attain such goal is the hierarchical clustering with complete link [11]. Using this algorithm, we can get a hierarchical tree, and then cut the tree into many $\beta$ coherent cliques according to certain choice of $\beta$. The definition of complete link ensures that the resulting clusters on cluster graph are $\beta$-coherent cliques.

\subsection{Profile and Pathway Extraction}

In section 2.2, we partition the entire cluster graph into many $\beta$-coherent cliques. In this section, we discuss how to robustly extract expression profile of the biological process underlying each coherent clique. We also address how to discover cellular pathways, i.e. set of coordinated genes, from expression profiles.

To begin with, recall that a coherent clique is composed of a set of nodes in cluster graph and each node refers to a cluster from a segment. Since each cluster covers certain conditions: the conditions covered by the segment where this cluster is generated, thus we can define the active period of a coherent clique:

- The active period $P(Q)$ of a coherent clique $Q$ is all the conditions that covered by at least one cluster in $Q$.

See figure 1 for an illustration: Supposed that coherent clique $Q$ is composed of three clusters, which are generated on segment $\mathrm{s}_{1}, \mathrm{~s}_{2}$ and $\mathrm{s}_{4}$, respectively. Then, the active period of $Q$ is $\left\{\mathrm{c}_{1}, \mathrm{c}_{2}, \mathrm{c}_{3}, \mathrm{c}_{4}, \mathrm{c}_{5}, \mathrm{c}_{6}, \mathrm{c}_{7}\right\}$.

Another important notion is the core genes:

- $\quad$ Gene $g$ is the core gene of coherent clique $Q$ if and only if $g$ is the member of all clusters in $Q$.

Furthermore, the expression profile involving the underlying biological process of coherent cluster $Q$ is:

- The expression profile of coherent cluster $Q$ is defined on $Q$ 's active period and computed as the mean expression of all the core genes of $\mathrm{Q}$.

Finally, we identify the cellular pathway corresponding to $Q$ based on its expression profile:

- A gene $g$ belongs to the cellular pathway of coherent clique $Q$ if and only if the Pearson correlation between $g$ 's expression and $Q$ 's expression profile on $Q$ 's active period exceeds 
$c$, the coherence parameter mentioned in section 2.1. Note that $g$ 's expression outside $Q$ 's active period is not considered.

\subsection{Further Analysis}

In this section we mainly discuss three properties: (1) ability to discover pathways on partial conditions; (2) identifying overlapping pathways; (3) robustness.

Firstly, if the active period of a biological process $P$ can be obtained by combining different segments produced in section 2.1, a corresponding coherent clique should be identified from cluster graph. This is based on two assumptions: (1) segment is defined in suitable granularity and highly overlapping; (2) genes cooperating in $P$ should co-express in $P$ 's active period.

Secondly, the overlapping property of pathways is ensured: (1) the active periods of diverse coherent cliques $Q_{1}$ and $Q_{2}$ differ, thus a gene $g$ can be consistent with both $Q_{l}$ 's expression profile in $Q_{l}$ 's active period and $Q_{2}$ 's expression profile in $Q_{2}$ 's active period. (2) Even on same period, expression level of gene $g$ can be consistent with different expression profiles.

Thirdly, the algorithm in this paper is robust, from four perspectives: (1) The definition of coherent clique is robust in that any two clusters in the clique have high overlapping degree and thus it is unlikely that an "outlier" cluster can be accommodated. (2) The computation of active period of each coherent clique is robust. See figure 1 for an illustration: supposed a biological process $P$ is active on conditions $\mathrm{c}_{1} \sim \mathrm{C}_{7}$, then the corresponding coherent clique $Q$ should have an active period as $\mathrm{c}_{1} \sim \mathrm{C}_{7}$. Ideally speaking $Q$ ought to consist of four clusters which are located in segments $\mathrm{s}_{1}$, $\mathrm{s}_{2}, \mathrm{~s}_{3}$ and $\mathrm{s}_{4}$, respectively, because $P$ is active on these segments and genes participate in $P$ should co-express on these segments. However, owing to high noise in microarrays, some clusters may be missed. As a result, $Q$ may consist of only three clusters on $\mathrm{s}_{1}, \mathrm{~s}_{2}$ and $\mathrm{s}_{4}$, respectively. Even in this case, the active period of $Q$ will be judged properly as $\mathrm{c}_{1} \sim \mathrm{C}_{7}$, based on $\mathrm{s}_{1}, \mathrm{~s}_{2}$ and $\mathrm{s}_{4}$. In short, segment overlapping ensures the robustness of active period estimation. (3) The choice of core genes in a coherent clique $Q$ is robust in that each core gene must belong to all the clusters in $Q$. Since these clusters are located in different segments and obtained by clustering on each segment independently, it is unlikely that an outlier gene will belong to all these clusters. Admittedly, this selection strategy is so "cautious" that it may miss some core genes. However, only a subset of core genes is still sufficient to extract expression profile of the underlying biological process, because core genes are supposed to co-express well in their biological process. (4) At last, the quality of core gene selection and active period estimation ensures the quality of expression profile extraction and the resultant pathway.

\section{EXPERIMENTAL RESULTS}

In this section we present empirical results. Compared with several state-of-the-art models, our algorithm is more capable of identifying biologically significant pathways in terms of the enrichment of gene functions.

\subsection{Dataset and Preprocessing}

Two well-know datasets used in our experiments are yeast cell cycle data [16] and yeast stress data [8]. For preprocessing, we remove genes with more than 5\% missing values and estimate missing values by KNNimpute [18]; then genes with small variance are removed. These steps result in 526 genes in cell cycle dataset and 659 genes in stress condition dataset.

\subsection{Rival Methods}

In this part, we introduce rival algorithms and their parameters: (1) HClust [7]: hierarchical clustering with average link and Pearson correlation. Finally 30 clusters are formed on both two datasets. (2) Plaid [12] is designed to discover biclusters one by one independently. The default parameters are used. We stop at 100 biclusters on both datasets. (3) OP [4] is a probabilistic model to search overlapping pathways simultaneously. The number of pathways is set as 30. (4) PIPE(Pathway Identification by Profile Extraction): our algorithm. The coherence threshold $c$ mentioned in section 2.1 and 2.3 is 0.7 ; the parameter $\beta$ used to define $\beta$-coherent clique is 0.7 . On cell cycle dataset which contains 76 conditions, we set segment length $L$ as 10 and step length $\Delta L=2$; for yeast stress dataset contains 173 conditions, $L$ is engaged as 20 and $\Delta L$ is set as 3 .

\subsection{Results on Cell Cycle}

Running our algorithm on 526 genes in 76 conditions results in 162 coherent cliques, thus finally we obtain 162 cellular pathways. Note that these pathways are generated independently, thus the fact that 162 


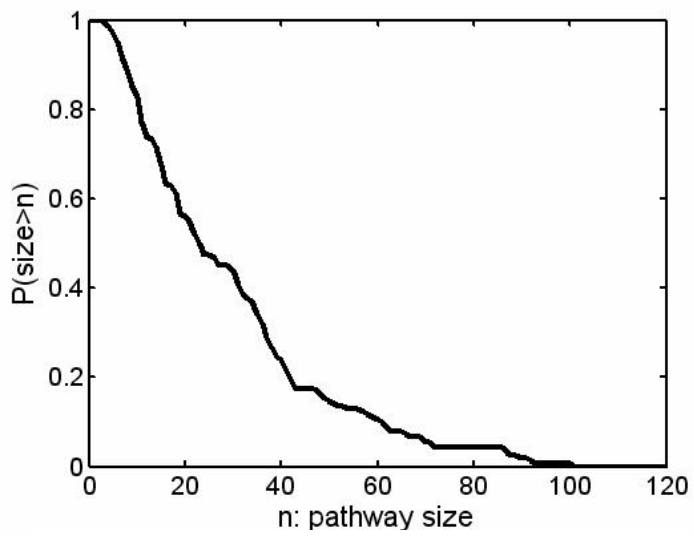

Fig. 2. Distribution of Pathway Size

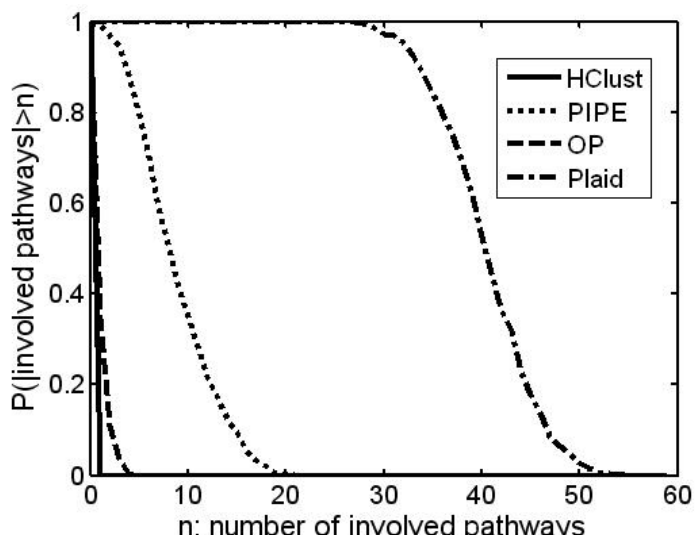

Fig. 3. Distribution of Gene Participation

pathways are produced from 526 genes does not indicate that the average size of pathways is quite small. In reality, the smallest pathway contains 4 genes and the largest one includes 101 genes. The distribution of pathway size is shown in figure 2. The $\mathrm{X}$-axis is pathway size; and the Y-axis is the proportion between pathways larger than specific size and all the pathways. From figure 2 we can observe that more than $80 \%$ pathways contain more than 10 genes, while only about $20 \%$ pathways contains more than 40 genes. This result shows that the majority of pathways have moderate size.

More interestingly, we measure the overlapping among pathways. HClust will certainly generate 30 mutually exclusive pathways, and running OP model results in 30 slightly overlapping pathways; at last, plaid model find 100 biclusters one by one. Figure 3 shows the distribution of amount of pathways that each gene participates in. The $\mathrm{X}$-axis is the number of pathways a gene joins; the $\mathrm{Y}$-axis is the proportion between the genes which involve more than a specific amount of pathways and all the genes. Four algorithms present quite different properties in figure 3: (1) Since HClust merely produce mutually exclusive pathways, all genes take part in only one pathway. (2) For OP model, single gene takes part in at most 5 pathways, and only 19 out of 526 genes participate in more than three pathways. (3) In Plaid algorithm, pathways are excessively overlapping: almost all genes participate in more than 30 pathways. (4) For our PIPE method, the result in figure 3 shows a natural distribution that only a few genes throw themselves into more than 15 pathways.

According to many researches concentrating on scale-free topology of biological networks [3] and especially of genetic regulatory networks [13]: (1) there should be a few "hub" genes connected with many other genes and thus join a lot of biological processes; (2) most genes in network should not have large degrees and thus they participate in only a few biological processes. As shown in figure 3, only our algorithm generates results consistent with above conclusion. HClust and OP model can not produce "hub" gene, and Plaid produce too many "hubs".

At last, to justify the biological significance of the pathways generated by these models, we test the enrichment of gene functions in GO categories [1]. For any pathway, the enrichment of a GO category is represented by p-value: the smaller the p-value, the better the enrichment. The p-value is computed based on Genomica [19]. For each GO category, we focus on the p-value of the pathway with best enrichment. For comparison, enrichment of all four algorithms are computed and listed in Table 1 . Note that p-value larger than 0.001 is considered as a failure to find enrichment and is labeled as “---” in the Table. Among 117 GO categories listed in Table 1: (1) PIPE won 70 times, while OP, HClust and Plaid models won 25, 21 and 1 times, respectively. (2) PIPE failed 23 times, yet OP, HClust and Plaid failed 41, 58 and 90 times, respectively. Further examining the results listed in Table 1 will naturally yield to the conclusion that PIPE has identified more biologically significant pathways.

\subsection{Results on Stress Condition}

To further justify the superiority of PIPE, we engage yeast stress condition dataset [8] for another experiment. Running our algorithm on 659 genes and 173 conditions brings about 174 pathways. Pathway size distribution of PIPE is demonstrated in figure 4, where a few pathways contain more than 100 genes and the majority are in 


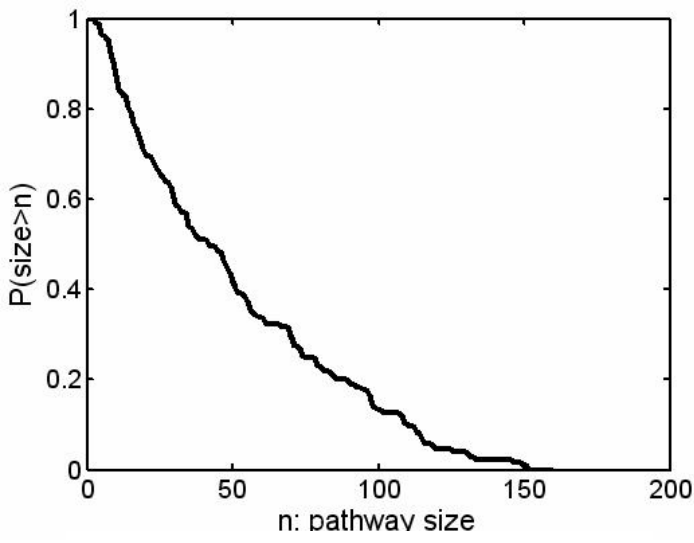

Fig. 4. Distribution of Pathway Size

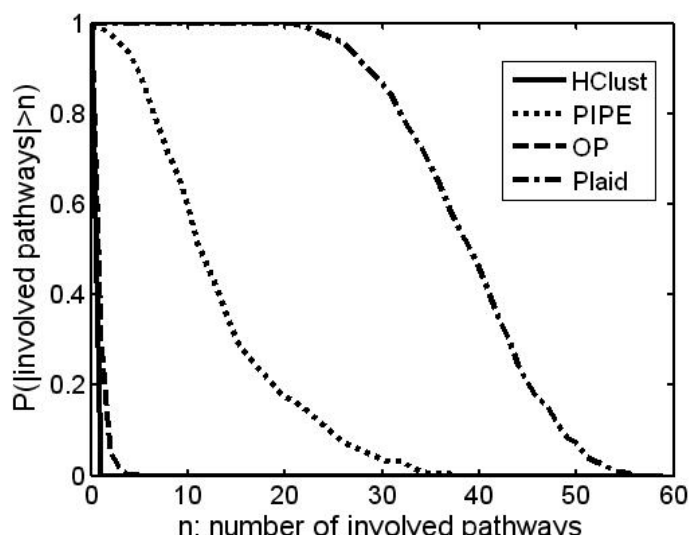

Fig. 5. Distribution of Gene Participation

moderate size. In addition, figure 5 shows similar results as observed in figure 3: HClust and OP can not discover any "hub" gene while Plaid considers most 659 genes as hubs that join a lot of pathways.

We also test the enrichment of GO categories and list the results in Table 2. From table 2 we can summarize that over 128 GO categories listed in Table2: (1) PIPE find best enrichment for 84 categories, while OP, HClust and Plaid find 18, 13 and 19 times, respectively. (2) PIPE failed to find enrichment for 11 GO terms, yet OP, HClust and Plaid failed 61, 81 and 68 times, respectively. In a word, PIPE has its own advantage to discover biologically meaningful pathways.

Another interesting fact is that Plaid algorithm performs much better on stress condition dataset than on cell cycle condition. One explanation for this result is the differences of regulation mechanism between endogenous phase (e.g. cell cycle and sporulation) and exogenous phase (e.g. stress condition, DNA damage and diauxic shift) [13]. In exogenous phase such as stress response, genes are often regulated by more transcriptional factor and participate in more processes than in endogenous phase such as cell cycle. Therefore, Plaid model, which tends to produce excessively overlapping pathways, results in more accurate results.

\section{CONCLUSION}

In this paper, we presented a new approach to discover cellular pathways. We firstly decompose gene expression matrix into highly overlapping segments and partition genes into clusters on each segment; then we organize all the resulting clusters into a cluster graph and identify coherent cliques; finally we extract expression profiles of coherent cliques and shape biological pathways from these profiles. We compare our algorithm with several recent models and the experimental results justify the superiorities of our approach: robustly identifying overlapping pathways on partial conditions and consequently discovering biologically significant pathways.

\section{Acknowledgments}

This work was supported in part by the National Science Foundation of China under Grant 60321002 and the Teaching and Research Award Program for Outstanding Young Teachers in Higher Education Institutions of MOE (TRAPOYT), China.

\section{References}

1 M. Ashburner, et al. Gene Ontology: tool for the unification of biology. Nat. Genet. 25: 25-29, 2000.

2 R. Balasubramaniyan, E. Hullermeier, N. Weskamp and J. Kamper. Clustering of gene expression data using a local shape-based similarity measure. Bioinformatics, 21(7): 1069-1077, 2005.

3 A.L. Barabasi and Z.N. Oltvai. Network biology: understanding the cell's functional organization. Nature Reviews Genetics, 5: 101, 2004.

4 A. Battle, E. Segal and D. Koller. Probabilistic discovery of overlapping cellular processes and their regulation. Journal of Computational Biology, 12(7): 909-927, 2005.

5 P.O. Brown and D. Bostein. Exploring the new world of the genome with DNA microarrays. Nat. Genet. 21, 33-37, 1999.

6 Y. Cheng and G.M. Church. Biclustering of expression data. ISMB, 2000.

7 M.B. Eisen, P.T. Spellman, P.O. Brown and D. Bostein. Cluster analysis and display of genome- 
wide expression patterns. PNAS. 95, 14863-14868, 1998.

8 A.P. Gasch, P.T. Spellman, C.M. Kao, O. CarmelHarel, M.B. Eisen, G. Storz, D. Botstein and P.O. Brown. Genomic expression programs in the response of yeast cells to environmental changes. Molecular Biology of the Cell, 11: 4241-4257, 2000.

9 T. Grotkjar, O. Winther, B. Regenberg, J. Nielsen and L.K. Hansen. Robust multi-scale clustering of large DNA microarray datasets with consensus algorithm. Bioinformatics, 22(1): 58-67, 2006.

10 D. Jiang, C. Tang and A. Zhang. Cluster analysis for gene expression data: a survey. IEEE trans. on Knowledge and Data Engineering, 16(11): 13791386, 2004.

11 B. King. Step-wise clustering procedures. J. Am. Stat. Assoc. 69, pages 86-101, 1967.

12 L. Lazzeroni and A. Owen. Plaid models for gene expression data. Technical report. Stanford Univ., 2000.

13 N.M. Luscombe, M.M. Babu, H. Yu, M. Snyder S.A. Teichmann and M. Gerstein. Genomic analysis of regulatory network dynamics reveals large topological changes. Nature, 431(7006): 308-
312, 2004

14 S.C. Madeira and A.L. Oliveira. Biclustering algorithms for biological data analysis: a survey. IEEE trans. on Computational Biology and Bioinformatics, 1(1): 24-45, 2004.

15 R. Sharan and R. Shamir. CLICK: a clustering algorithm with applications to gene expression analysis. ISMB, 2000.

16 P.T. Spellman, G. Sherlock, M.Q. Zhang, V.R. Iyer, K. Anders, M.B. Eisen, P.Q. Brown, D. Botstein and B. Futcher. Comprehensive identification of cell cycle-regulated genes of the yeast Saccharomyces Cerevisiae by microarray hybridization. Molecular Biology of the Cell, 9: 3273-3297, 1998.

17 S. Tavazoie, J.D. Hughes, M.J. Campbell, R.J. Cho and G.M. Church. Systematic determination of genetic network architecture. Nat. Genet. 22, 281285, 1999.

18 O. Troyanskaya, M. Cantor, G. Sherlock, P. Brown, T. Hastie, R. Tibshirani, D. Botstein and R.B. Altman. Missing value estimation methods for DNA microarrays. Bioinformatics, 17: 520-525, 2001.

19 http://genomica.weizmann.ac.il/index.html

Table 1. GO Categories Enrichment based on Cell Cycle Dataset

\begin{tabular}{|c|c|c|c|c|}
\hline GO term & HClust & PIPE & OP & Plaid \\
\hline 35S Primary Transcript Processing & 4.01E-6 & 1.05E-7 & $3.55 \mathrm{E}-5$ & --- \\
\hline amine biosynthesis & --- & 1.91E-6 & 7.02E-6 & --- \\
\hline amine metabolism & --- & $2.48 \mathrm{E}-6$ & 2.45E-6 & --- \\
\hline amine transport & $7.56 \mathrm{E}-5$ & --- & --- & --- \\
\hline aspartate family amino acid metabolism & --- & --- & 1.10E-7 & --- \\
\hline ATP dependent DNA helicase activity & --- & 2.49E-6 & --- & --- \\
\hline ATP dependent helicase activity & --- & 2.14E-5 & --- & --- \\
\hline beta-glucosidase activity & --- & --- & 5.77E-6 & --- \\
\hline bud neck & --- & 5.29E-6 & --- & --- \\
\hline carbohydrate metabolism & --- & 1.25E-4 & --- & --- \\
\hline carbohydrate transport & 1.70E-7 & $3.20 \mathrm{E}-5$ & 2.57E-6 & $6.88 \mathrm{E}-5$ \\
\hline carrier activity & --- & $2.58 \mathrm{E}-5$ & 2.84E-4 & --- \\
\hline cation transport & --- & 2.57E-4 & --- & $2.60 \mathrm{E}-5$ \\
\hline cation transporter activity & --- & $7.60 \mathrm{E}-5$ & 2.16E-4 & $1.98 \mathrm{E}-4$ \\
\hline cell communication & --- & 2.79E-5 & --- & --- \\
\hline cell wall & 7.94E-6 & --- & 2.89E-8 & $3.06 \mathrm{E}-5$ \\
\hline chromatin assembly or disassembly & 4.66E-7 & 8.15E-11 & 3.49E-7 & --- \\
\hline chromatin binding & 3.95E-5 & 4.33E-7 & 3.95E-5 & --- \\
\hline conjugation & --- & 4.17E-5 & --- & --- \\
\hline contractile ring & 3.95E-5 & 8.45E-7 & 8.82E-5 & --- \\
\hline
\end{tabular}




\begin{tabular}{|c|c|c|c|c|}
\hline cyclin-dependent protein kinase regular activity & --- & 9.09E-5 & --- & --- \\
\hline cytokinesis & $2.12 \mathrm{E}-4$ & 4.59E-6 & --- & --- \\
\hline cytokinesis, completion of separation & $1.30 \mathrm{E}-5$ & $1.97 \mathrm{E}-5$ & 1.11E-8 & --- \\
\hline cytoplasmic vesicle & --- & --- & 2.82E-5 & --- \\
\hline cytoskeleton organization and biogenesis & --- & 1.05E-4 & --- & --- \\
\hline cytosolic large ribosomal subnit (sensu Eukaryota) & $2.86 \mathrm{E}-10$ & 1.18E-10 & $6.26 \mathrm{E}-10$ & --- \\
\hline cytosolic small ribosomal subnit (sensu Eukaryota) & $2.19 \mathrm{E}-5$ & $1.39 \mathrm{E}-5$ & $3.29 \mathrm{E}-5$ & --- \\
\hline development & --- & 1.37E-4 & --- & --- \\
\hline DNA binding & $1.77 \mathrm{E}-4$ & 2.45E-8 & $2.26 \mathrm{E}-6$ & $2.87 \mathrm{E}-6$ \\
\hline DNA helicase activity & --- & 1.04E-6 & $3.74 \mathrm{E}-5$ & --- \\
\hline DNA metabolism & 4.36E-9 & 3.25E-8 & $2.82 \mathrm{E}-7$ & $1.88 \mathrm{E}-5$ \\
\hline DNA packaging & $3.74 \mathrm{E}-5$ & $2.24 \mathrm{E}-8$ & $1.80 \mathrm{E}-4$ & --- \\
\hline DNA recombination & 7.99E-6 & 4.45E-10 & $5.84 \mathrm{E}-5$ & --- \\
\hline DNA repair & $1.62 \mathrm{E}-6$ & 8.37E-8 & $2.56 \mathrm{E}-5$ & $1.98 \mathrm{E}-4$ \\
\hline DNA replication & --- & 1.18E-4 & --- & --- \\
\hline DNA replication initiation & --- & 2.14E-5 & $5.84 \mathrm{E}-5$ & --- \\
\hline DNA replication, synthesis, of RNA primer & --- & 2.71E-4 & --- & --- \\
\hline DNA strand elongation & 1.21E-6 & 4.12E-6 & $1.20 \mathrm{E}-5$ & 1.07E-4 \\
\hline DNA unwinding & $2.12 \mathrm{E}-4$ & 2.49E-6 & $2.12 \mathrm{E}-4$ & --- \\
\hline DNA-dependent ATPase activity & $2.12 \mathrm{E}-4$ & 2.49E-6 & $2.12 \mathrm{E}-4$ & --- \\
\hline DNA-directed DNA polymerase activity & $6.00 \mathrm{E}-5$ & 9.86E-7 & $2.53 \mathrm{E}-4$ & --- \\
\hline electron transporter activity & $2.26 \mathrm{E}-4$ & --- & 1.36E-4 & --- \\
\hline endoplasmic reticulum & --- & $1.96 \mathrm{E}-4$ & --- & --- \\
\hline endosome & --- & --- & 2.82E-5 & --- \\
\hline energy pathways & 2.84E-6 & 1.33E-4 & $3.05 \mathrm{E}-5$ & --- \\
\hline glucosidase activity & --- & --- & 7.63E-7 & --- \\
\hline glutamate metabolism & 4.13E-6 & 9.92E-6 & $1.20 \mathrm{E}-4$ & --- \\
\hline glutamine family amino acid biosynthesis & 4.13E-6 & 9.92E-6 & $1.20 \mathrm{E}-4$ & --- \\
\hline glutamine family amino acid metabolism & 3.52E-5 & 8.28E-5 & --- & --- \\
\hline helicase activity & --- & 5.51E-6 & --- & --- \\
\hline hexose transport & $2.10 \mathrm{E}-8$ & 7.02E-6 & $3.26 \mathrm{E}-7$ & --- \\
\hline hydrolase activity & --- & --- & 1.16E-4 & --- \\
\hline ion transporter activity & --- & $1.10 \mathrm{E}-4$ & 3.16E-5 & --- \\
\hline iron ion transport & --- & --- & 2.82E-5 & --- \\
\hline iron ion transporter activity & --- & --- & 7.55E-7 & --- \\
\hline iron-siderochrome transport & --- & --- & 2.82E-5 & --- \\
\hline kinase inhibitor activity & --- & --- & $1.86 \mathrm{E}-4$ & --- \\
\hline lagging strand elongation & --- & 5.63E-6 & --- & --- \\
\hline large ribosomal subunit & $1.26 \mathrm{E}-9$ & $5.20 \mathrm{E}-10$ & 2.75E-9 & $2.42 \mathrm{E}-4$ \\
\hline leading strand elongation & --- & 2.71E-4 & --- & --- \\
\hline main pathways of carbohydrate metabolism & 2.84E-6 & $1.76 \mathrm{E}-4$ & 4.63E-5 & --- \\
\hline mannose transporter activity & $2.10 \mathrm{E}-8$ & 7.02E-6 & $3.26 \mathrm{E}-7$ & --- \\
\hline MCM complex & $3.95 \mathrm{E}-5$ & 4.33E-7 & $3.95 \mathrm{E}-5$ & --- \\
\hline metal ion transporter activity & --- & --- & 2.49E-5 & --- \\
\hline methionine metabolism & 8.59E-5 & --- & 2.22E-8 & --- \\
\hline mismatch repair & $8.29 \mathrm{E}-6$ & 1.42E-6 & $4.71 \mathrm{E}-5$ & \\
\hline
\end{tabular}




\begin{tabular}{|c|c|c|c|c|}
\hline mitochondrion & --- & 1.31E-4 & --- & $2.76 \mathrm{E}-4$ \\
\hline mitotic recombination & $8.29 \mathrm{E}-6$ & 8.34E-9 & $4.71 \mathrm{E}-5$ & --- \\
\hline nuclear organization and biogenesis & --- & $1.48 \mathrm{E}-7$ & --- & --- \\
\hline nucleic acid binding & --- & 1.57E-7 & $4.52 \mathrm{E}-5$ & $1.93 \mathrm{E}-6$ \\
\hline nucleolus & 8.01E-23 & $7.10 \mathrm{E}-20$ & $2.42 \mathrm{E}-18$ & 4.90E-9 \\
\hline nucleosome & 5.46E-09 & 1.35E-13 & 3.90E-9 & --- \\
\hline nucleotide-excision repair & --- & 1.66E-5 & --- & --- \\
\hline nucleotidyltransferase activity & --- & 4.76E-5 & --- & --- \\
\hline organic acid metabolism & --- & $9.04 \mathrm{E}-5$ & $1.80 \mathrm{E}-5$ & --- \\
\hline polyamine transport & --- & $9.98 \mathrm{E}-6$ & --- & --- \\
\hline pre-replicative complex & --- & $1.40 \mathrm{E}-7$ & $3.13 \mathrm{E}-5$ & --- \\
\hline pre-replicative complex formation and maintenance & --- & $1.40 \mathrm{E}-7$ & $3.13 E-5$ & --- \\
\hline protein amino acid glycosylation & $1.42 \mathrm{E}-4$ & --- & --- & --- \\
\hline protein binding & $1.54 \mathrm{E}-5$ & 4.69E-5 & --- & 1.63E-4 \\
\hline protein biosynthesis & $5.80 \mathrm{E}-13$ & 1.13E-13 & $2.36 \mathrm{E}-12$ & 8.46E-6 \\
\hline protein folding & 2.17E-12 & $2.16 \mathrm{E}-10$ & $1.07 \mathrm{E}-10$ & --- \\
\hline pyrophosphatase activity & --- & 2.08E-4 & --- & --- \\
\hline regulation of cell cycle & --- & 3.51E-5 & --- & --- \\
\hline regulation of cyclin dependent protein kinase activity & --- & $1.18 \mathrm{E}-4$ & --- & --- \\
\hline regulation of enzyme activity & --- & $1.18 \mathrm{E}-4$ & --- & --- \\
\hline regulation of metabolism & --- & 3.46E-5 & --- & --- \\
\hline regulation of transcription & --- & $1.76 \mathrm{E}-5$ & --- & --- \\
\hline regulation of transcription from Pol 2 promoter & --- & 3.06E-5 & --- & --- \\
\hline replication fork & --- & --- & 4.71E-05 & --- \\
\hline response to DNA damage stimulus & 4.43E-9 & $7.21 \mathrm{E}-9$ & $2.41 \mathrm{E}-7$ & 2.12E-5 \\
\hline response to stimulus & $2.46 \mathrm{E}-5$ & 8.37E-6 & $2.04 \mathrm{E}-4$ & --- \\
\hline response to stress & 4.67E-5 & 1.15E-5 & --- & --- \\
\hline ribosomal large subunit biogenesis & $9.54 \mathrm{E}-5$ & 6.84E-6 & --- & --- \\
\hline ribosomal small subunit assemble and maintenance & --- & $1.86 \mathrm{E}-4$ & --- & --- \\
\hline ribosomal subunit assembly & --- & $9.98 \mathrm{E}-6$ & --- & --- \\
\hline RNA binding & $2.46 \mathrm{E}-4$ & --- & --- & --- \\
\hline RNA metabolism & $2.46 \mathrm{E}-12$ & $2.61 \mathrm{E}-11$ & $1.96 \mathrm{E}-9$ & 1.07E-4 \\
\hline RNA processing & $2.10 \mathrm{E}-12$ & $1.50 \mathrm{E}-12$ & 8.62E-10 & $1.46 \mathrm{E}-6$ \\
\hline rRNA processing & 8.54E-14 & $1.95 \mathrm{E}-13$ & 3.73E-11 & $1.20 \mathrm{E}-5$ \\
\hline siderochrome transport & $1.65 \mathrm{E}-6$ & $9.94 \mathrm{E}-5$ & $1.88 E-8$ & --- \\
\hline site of polarized growth & --- & 2.36E-4 & --- & --- \\
\hline small ribosomal sununit & $2.19 \mathrm{E}-5$ & $1.39 \mathrm{E}-5$ & $3.29 \mathrm{E}-5$ & --- \\
\hline structural constituent of ribosome & $1.12 \mathrm{E}-14$ & 2.15E-15 & 4.63E-14 & 8.24E-7 \\
\hline sulfur amino acid biosynthesis & 8.59E-5 & --- & 2.22E-8 & --- \\
\hline sulfur amino acid metabolism & 7.89E-6 & --- & $1.70 \mathrm{E}-10$ & --- \\
\hline sulfur metabolism & $1.42 \mathrm{E}-4$ & --- & 3.54E-9 & --- \\
\hline telomerase-independent telomere maintenance & $6.00 \mathrm{E}-5$ & $5.18 \mathrm{E}-7$ & $2.53 \mathrm{E}-4$ & --- \\
\hline telomere maintenance & $6.00 \mathrm{E}-5$ & $5.18 \mathrm{E}-7$ & $2.53 \mathrm{E}-4$ & --- \\
\hline transcription regulator activity & --- & $2.78 \mathrm{E}-4$ & --- & --- \\
\hline transferase activity and phosphorus-contain group & --- & 2.04E-4 & --- & --- \\
\hline transcription metal ion transporter activity & --- & --- & $2.49 E-5$ & --- \\
\hline
\end{tabular}




\begin{tabular}{|l|l|l|l|l|}
\hline translational elongation & $1.54 \mathrm{E}-6$ & $\mathbf{9 . 7 0 E}-7$ & $2.32 \mathrm{E}-6$ & --- \\
\hline tricarboxylic acid cycle & $2.26 \mathrm{E}-4$ & --- & $\mathbf{1 . 6 8 E}-\mathbf{4}$ & --- \\
\hline tricarboxylic acid cycle intermediate metabolism & $\mathbf{7 . 1 7 E}-7$ & $1.23 \mathrm{E}-4$ & $4.17 \mathrm{E}-5$ & --- \\
\hline unfolded protein binding & $\mathbf{1 . 6 8 E - 9}$ & $4.21 \mathrm{E}-7$ & $4.14 \mathrm{E}-8$ & $2.94 \mathrm{E}-5$ \\
\hline vesicle-mediated transport & --- & $\mathbf{1 . 1 1 E}-4$ & --- & --- \\
\hline
\end{tabular}

Table 2. GO Categories Enrichment based on Stress Condition Dataset

\begin{tabular}{|c|c|c|c|c|}
\hline GO term & HClust & PIPE & OP & Plaid \\
\hline 35S Primary Transcript Processing & $3.11 \mathrm{E}-7$ & 4.27E-10 & $6.35 \mathrm{E}-7$ & $3.46 \mathrm{E}-9$ \\
\hline acid phosphatase activity & --- & $1.18 \mathrm{E}-6$ & 4.21E-7 & --- \\
\hline aerobic respiration & --- & 3.74E-6 & --- & --- \\
\hline alcohol metabolism & $7.70 \mathrm{E}-5$ & $1.48 \mathrm{E}-5$ & $7.70 \mathrm{E}-5$ & $8.57 \mathrm{E}-5$ \\
\hline amine biosynthesis & --- & 8.56E-10 & $2.54 \mathrm{E}-4$ & $1.27 \mathrm{E}-9$ \\
\hline amine metabolism & $4.94 \mathrm{E}-4$ & 1.73E-12 & 4.93E-4 & 2.41E-15 \\
\hline amine transport activity & 2.01E-4 & --- & 3.17E-4 & --- \\
\hline aspartate family amino acid biosynthesis & --- & 1.31E-5 & --- & $5.08 \mathrm{E}-4$ \\
\hline aspartate family amino acid metabolism & $9.58 \mathrm{E}-6$ & 3.02E-10 & $9.58 \mathrm{E}-6$ & $3.44 \mathrm{E}-5$ \\
\hline ATP dependent DNA helicase activity & --- & 2.13E-5 & --- & $1.64 \mathrm{E}-4$ \\
\hline carbohydrate catabolism & --- & --- & 2.41E-4 & --- \\
\hline carbohydrate kinase activity & --- & 3.03E-5 & --- & --- \\
\hline carbohydrate metabolism & $6.28 \mathrm{E}-10$ & 5.67E-9 & $9.50 \mathrm{E}-7$ & 2.71E-10 \\
\hline carbohydrate transport & --- & 6.67E-7 & $2.10 \mathrm{E}-4$ & --- \\
\hline cell communication & --- & $2.06 E-4$ & --- & --- \\
\hline cell wall & --- & 1.72E-7 & $1.77 \mathrm{E}-6$ & 4.95E-4 \\
\hline cytosolic large ribosomal subnit (sensu Eukaryota) & $1.65 \mathrm{E}-19$ & 1.50E-32 & $1.35 \mathrm{E}-18$ & $4.18 \mathrm{E}-20$ \\
\hline cytosolic small ribosomal subnit (sensu Eukaryota) & $2.02 \mathrm{E}-11$ & 2.36E-21 & $6.56 \mathrm{E}-11$ & 4.33E-12 \\
\hline disaccharide metabolism & --- & 4.26E-5 & --- & --- \\
\hline DNA binding & --- & 1.55E-4 & --- & --- \\
\hline DNA-directed RNA polymerase 1 complex & --- & $2.92 E-4$ & --- & --- \\
\hline endoplasmic reticulum & --- & $2.86 \mathrm{E}-5$ & $2.34 \mathrm{E}-5$ & --- \\
\hline energy pathways & $1.87 \mathrm{E}-12$ & $1.64 \mathrm{E}-10$ & $5.30 \mathrm{E}-14$ & 9.49E-14 \\
\hline energy reserve metabolism & $9.12 \mathrm{E}-5$ & 3.49E-6 & 3.03E-5 & 3.55E-4 \\
\hline enzyme regulator activity & --- & 1.74E-4 & --- & --- \\
\hline galactose metabolism & 2.11E-8 & $2.11 \mathrm{E}-7$ & 2.11E-8 & --- \\
\hline glucan metabolism & --- & 5.67E-5 & $6.53 \mathrm{E}-5$ & --- \\
\hline glucose metabolism & --- & --- & --- & 8.08E-5 \\
\hline glucosyltransferase activity & --- & 3.42E-4 & --- & --- \\
\hline glutamate metabolism & --- & 9.34E-5 & --- & --- \\
\hline glutamine family amino acid biosynthesis & --- & $3.78 \mathrm{E}-7$ & --- & $2.26 \mathrm{E}-4$ \\
\hline glutamine family amino acid metabolism & --- & $5.55 E-6$ & --- & --- \\
\hline glutathione peroxidase activity & --- & $1.38 \mathrm{E}-4$ & --- & --- \\
\hline glycogen metabolism & --- & $5.67 E-5$ & $6.53 \mathrm{E}-5$ & --- \\
\hline helicase activity & $4.26 \mathrm{E}-4$ & $9.89 \mathrm{E}-7$ & --- & $1.39 \mathrm{E}-4$ \\
\hline heterocycle metabolism & $4.72 \mathrm{E}-4$ & 1.43E-4 & --- & --- \\
\hline hexose metabolism & $1.43 E-5$ & $1.39 \mathrm{E}-4$ & $1.43 E-5$ & --- \\
\hline
\end{tabular}




\begin{tabular}{|c|c|c|c|c|}
\hline hexose transport & --- & $9.66 \mathrm{E}-7$ & $6.53 \mathrm{E}-5$ & --- \\
\hline hexose transporter activity & --- & $1.46 \mathrm{E}-4$ & --- & --- \\
\hline hydrolase activity acting on ester bonds & --- & --- & 2.73E-4 & --- \\
\hline ion transporter activity & --- & 2.37E-4 & --- & --- \\
\hline kinase activity & $9.12 \mathrm{E}-5$ & 9.79E-6 & --- & --- \\
\hline large ribosomal subunit & 1.65E-19 & $1.50 \mathrm{E}-32$ & 1.35E-18 & 4.18E-20 \\
\hline ligase activity, forming carbon-nitrogen bonds & --- & --- & --- & 4.47E-4 \\
\hline lipid metabolism & --- & --- & --- & 4.19E-4 \\
\hline main pathways of carbohydrate metabolism & 3.59E-4 & $2.51 \mathrm{E}-6$ & $5.29 \mathrm{E}-8$ & $7.70 \mathrm{E}-6$ \\
\hline mannose transporter activity & --- & 3.64E-4 & --- & --- \\
\hline methionine metabolism & $1.18 \mathrm{E}-6$ & 4.47E-7 & $1.18 \mathrm{E}-6$ & --- \\
\hline methyltransferase activity & --- & 1.24E-5 & --- & $8.48 \mathrm{E}-5$ \\
\hline mitochondrion & --- & 4.83E-5 & 4.40E-4 & 2.51E-7 \\
\hline Noc complex & --- & 2.25E-4 & --- & --- \\
\hline non-membrane-bound organelle & --- & 1.73E-4 & --- & --- \\
\hline nucleic acid binding & 9.82E-12 & $6.29 \mathrm{E}-8$ & $6.44 \mathrm{E}-11$ & 2.17E-11 \\
\hline nucleolus & $6.66 \mathrm{E}-21$ & 7.55E-26 & $6.73 \mathrm{E}-20$ & $1.51 \mathrm{E}-21$ \\
\hline nucleotide biosynthesis & $1.74 \mathrm{E}-5$ & $1.74 \mathrm{E}-5$ & $2.76 \mathrm{E}-4$ & --- \\
\hline nucleotide metabolism & 1.14E-4 & 1.14E-4 & --- & --- \\
\hline organic acid metabolism & --- & $1.72 \mathrm{E}-10$ & 2.73E-4 & 1.33E-13 \\
\hline oxidoreductase activity & 4.65E-4 & $1.17 \mathrm{E}-5$ & $2.73 \mathrm{E}-4$ & 1.85E-6 \\
\hline oridoreductase activity on $\mathrm{CH}-\mathrm{OH}$ group of donors & --- & 6.02E-5 & --- & 3.33E-4 \\
\hline pentose metabolism & --- & 1.93E-4 & 6.26E-5 & --- \\
\hline peroxidase activity & --- & 1.16E-9 & $1.34 \mathrm{E}-7$ & 7.03E-5 \\
\hline peroxisomal matrix & --- & --- & 3.76E-4 & --- \\
\hline phosphoric ester hydrolase activity & --- & $3.18 \mathrm{E}-4$ & 1.16E-4 & --- \\
\hline polysaccharide metabolism & --- & $1.28 \mathrm{E}-4$ & $2.10 \mathrm{E}-4$ & --- \\
\hline processing of 20S pre-rRNA & 1.79E-9 & $7.70 \mathrm{E}-11$ & 4.69E-9 & 4.39E-11 \\
\hline protein binding & --- & $1.74 \mathrm{E}-7$ & 3.44E-11 & $2.52 \mathrm{E}-4$ \\
\hline protein biosynthesis & 2.82E-29 & 3.41E-60 & $1.64 \mathrm{E}-27$ & $1.82 \mathrm{E}-27$ \\
\hline protein folding & $2.36 \mathrm{E}-7$ & $1.95 \mathrm{E}-15$ & $6.67 \mathrm{E}-17$ & $2.61 \mathrm{E}-8$ \\
\hline purine nucleotide metabolism & $1.74 \mathrm{E}-5$ & $1.74 \mathrm{E}-5$ & $2.76 \mathrm{E}-4$ & --- \\
\hline pyrophosphatase activity & --- & $3.55 \mathrm{E}-4$ & --- & 3.51E-5 \\
\hline regulation of biosynthesis & --- & 2.85E-6 & --- & --- \\
\hline regulation of catabolism & --- & $1.38 \mathrm{E}-4$ & --- & --- \\
\hline regulation of cell redox homeostasis & --- & $1.34 \mathrm{E}-7$ & --- & --- \\
\hline regulation of cellular process & --- & 2.61E-7 & --- & --- \\
\hline regulation of translation & --- & 6.97E-8 & --- & --- \\
\hline regulation of translational fidelity & --- & 1.33E-6 & --- & --- \\
\hline respiratory chain complex 3 & --- & 4.72E-5 & $1.18 \mathrm{E}-4$ & --- \\
\hline response to biotic stimulus & --- & 8.64E-5 & --- & $5.28 \mathrm{E}-5$ \\
\hline response to osmotic stress & --- & --- & 1.74E-5 & --- \\
\hline response to stimulus & --- & $1.29 \mathrm{E}-5$ & 4.22E-8 & $6.96 \mathrm{E}-11$ \\
\hline response to stress & --- & 2.62E-6 & 8.32E-9 & $6.39 \mathrm{E}-12$ \\
\hline ribonucleoprotein complex & 3.74E-9 & 8.38E-8 & $1.14 \mathrm{E}-8$ & $2.44 \mathrm{E}-8$ \\
\hline ribosomal large sununit assembly and maintenance & $1.34 \mathrm{E}-6$ & $1.24 \mathrm{E}-5$ & $2.54 \mathrm{E}-6$ & 8.76E-7 \\
\hline
\end{tabular}




\begin{tabular}{|c|c|c|c|c|}
\hline ribosomal large subunit biogenesis & --- & 4.22E-6 & --- & $4.92 \mathrm{E}-4$ \\
\hline ribosome & --- & 6.72E-5 & --- & --- \\
\hline ribosome biogenesis & 4.97E-5 & $1.04 \mathrm{E}-4$ & 3.65E-5 & 7.95E-5 \\
\hline RNA binding & 3.75E-14 & $5.21 \mathrm{E}-11$ & $2.09 \mathrm{E}-13$ & $2.54 \mathrm{E}-13$ \\
\hline RNA helicase activity & $4.26 \mathrm{E}-4$ & $9.89 \mathrm{E}-7$ & --- & $1.38 \mathrm{E}-4$ \\
\hline RNA ligase activity & 4.97E-5 & 8.23E-10 & 7.95E-5 & $2.22 \mathrm{E}-7$ \\
\hline RNA metabolism & $5.78 \mathrm{E}-30$ & 2.02E-20 & 5.09E-27 & $1.60 \mathrm{E}-28$ \\
\hline RNA methyltransferase activity & --- & 1.19E-4 & --- & --- \\
\hline RNA modification & $7.88 \mathrm{E}-9$ & $3.03 E-8$ & $1.92 \mathrm{E}-8$ & $1.22 \mathrm{E}-7$ \\
\hline RNA polymerase complex & --- & $2.92 E-4$ & --- & --- \\
\hline RNA processing & 2.59E-22 & $3.68 \mathrm{E}-22$ & $2.92 \mathrm{E}-21$ & 5.29E-23 \\
\hline RNA-dependent ATPase activity & --- & 2.13E-5 & --- & $1.64 \mathrm{E}-4$ \\
\hline rRNA binding & --- & $2.89 \mathrm{E}-5$ & --- & $1.64 \mathrm{E}-4$ \\
\hline rRNA modification & --- & $3.41 E-6$ & --- & 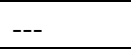 \\
\hline rRNA processing & $2.59 \mathrm{E}-22$ & $3.68 \mathrm{E}-22$ & $2.92 \mathrm{E}-21$ & $5.29 E-23$ \\
\hline S-adenosylmethionine-dependent methyltransferease & --- & --- & --- & 4.46E-4 \\
\hline signal transduction & --- & 9.34E-5 & --- & --- \\
\hline small nuclear ribonucleoprotein complex & --- & 1.73E-4 & --- & --- \\
\hline small nucleolar ribonucleoprotein complex & $1.50 \mathrm{E}-7$ & $1.08 \mathrm{E}-7$ & 3.17E-7 & 1.16E-9 \\
\hline small ribosomal sununit & 2.01E-11 & 2.32E-21 & $6.56 \mathrm{E}-11$ & 4.33E-12 \\
\hline snoRNA binding & $3.11 \mathrm{E}-7$ & $2.10 \mathrm{E}-8$ & $6.53 \mathrm{E}-7$ & 7.56E-11 \\
\hline SRP-dependent protein-membrane target & --- & --- & $5.48 E-5$ & --- \\
\hline structural constituent of ribosome & 2.29E-29 & 2.55E-61 & 7.00E-28 & 7.76E-31 \\
\hline succinate dehydrogenase (ubiquinone) activity & --- & 7.03E-6 & --- & --- \\
\hline sulfur amino acid metabolism & 3.48E-6 & 3.48E-6 & 3.48E-6 & $1.45 \mathrm{E}-4$ \\
\hline sulfur metabolism & 7.67E-6 & $1.45 \mathrm{E}-5$ & 7.67E-6 & --- \\
\hline thioredoxin peroxidase activity & --- & 6.72E-6 & --- & --- \\
\hline transcription from Pol 1 promoter & --- & 2.92E-4 & --- & --- \\
\hline transcription regulator activity & --- & $1.55 E-4$ & --- & --- \\
\hline transcription, DNA-dependent & --- & $2.92 E-4$ & --- & --- \\
\hline transferase activity, transferase acyl groups & --- & $3.23 E-5$ & --- & --- \\
\hline transferase activity and phosphorus-contain group & --- & $3.60 \mathrm{E}-4$ & --- & --- \\
\hline transcription factor activity, nucleic acid binding & --- & $5.01 E-6$ & --- & --- \\
\hline transcription initiation factor activity & --- & 1.44E-4 & --- & --- \\
\hline transcription regulator activity & --- & 5.01E-6 & --- & --- \\
\hline translational elongation & --- & 8.46E-7 & --- & --- \\
\hline trehalose metabolism & --- & $4.26 \mathrm{E}-5$ & --- & --- \\
\hline tricarboxylic acid cycle & --- & $1.26 \mathrm{E}-8$ & $2.77 \mathrm{E}-5$ & --- \\
\hline tricarboxylic acid cycle intermediate metabolism & --- & --- & $7.61 E-5$ & $3.55 E-4$ \\
\hline tRNA aminoacylation & $2.09 \mathrm{E}-4$ & 2.81E-8 & $3.12 \mathrm{E}-4$ & $2.06 \mathrm{E}-6$ \\
\hline tRNA metabolism & $2.76 \mathrm{E}-6$ & $3.80 \mathrm{E}-8$ & $5.08 \mathrm{E}-6$ & $1.75 \mathrm{E}-5$ \\
\hline tRNA methyltransferase activity & --- & 6.85E-5 & --- & --- \\
\hline tRNA modification & $2.76 \mathrm{E}-6$ & $3.80 \mathrm{E}-8$ & $5.08 \mathrm{E}-6$ & $1.79 \mathrm{E}-5$ \\
\hline UDP-glycosyltransferase activity & --- & $3.42 E-4$ & --- & --- \\
\hline unfolded protein binding & $3.66 \mathrm{E}-4$ & $1.87 \mathrm{E}-7$ & 1.05E-11 & $1.14 \mathrm{E}-4$ \\
\hline
\end{tabular}

